Eva Wiesmann*

\title{
Zum Wissen des Rechtsübersetzers und zur Wissensaufbereitung in übersetzerischen Hilfsmitteln
}

\begin{abstract}
This article deals with the difficulties encountered by the translator with no legal background in consulting resources conveying legal knowledge, ranging from the text of the law to the legal encyclopaedia, designed for lawyers, lawyers and non-lawyers, lawyers and legal translators respectively. Starting from the translation from Italian into German of a text of company law, there is first of all a description of the legal knowledge required by the legal translator to translate the legal text in question and the legal knowledge conveyed by the text for translation itself. After an overview of the resources that the legal translator may draw on to integrate any gaps in his legal knowledge, there is an illustration of the problems linked to the different types of resources, concluding with a demonstration of the advantages of a resource such as JUSLEX, designed and created exclusively for the legal translator.
\end{abstract}

\section{1. $\quad$ Einleitung}

Gegenstand dieses Beitrags sind die Probleme, die der nicht juristisch geschulte Rechtsübersetzer beim Umgang mit den rechtliches Wissen vermittelnden Hilfsmitteln hat, die a) an Juristen, b) an Juristen und Nichtjuristen oder c) an Juristen und Rechtsübersetzer adressiert sind. Dabei wird zunächst herausgearbeitet, welches rechtliche Wissen der Rechtsübersetzer zur Übersetzung eines gegebenen juristischen Textes benötigt, welches Wissen er dem Text selbst entnehmen kann und welche übersetzerischen Hilfsmittel ihm zur Verfügung stehen, um das ihm fehlende Wissen zu ergänzen. Abschließend wird aufgezeigt, inwieweit

\footnotetext{
* Eva Wiesmann

Alma Mater Studiorum

Università di Bologna - sede di Forlì

Dipartimento di Studi Interdisciplinari su Traduzione, Lingue e Culture

Corso A. Diaz 64

I-47100 Forlì (FC)

wiesmann@sslmit.unibo.it
}

Hermes - Journal of Language and Communication Studies no 40-2008 
die allein für den Rechtsübersetzer entworfene und entwickelte Datenbank JUSLEX Abhilfe schaffen kann.

\section{Beispieltext und Übersetzung}

Bei dem zu übersetzenden Text handelt es sich um einen Abschnitt aus dem Artikel Regole in autonomia (Eigene Normen für die italienische Gesellschaft mit beschränkter Haftung; im Folgenden kurz ,italienische $\mathrm{GmbH}^{\star}$ oder ,Srl`, d.h. società a responsabilità limitata), der die gesetzlichen Neuerungen betreffend die unbeschränkte Haftung des Alleingesellschafters der italienischen $\mathrm{GmbH}$ zum Gegenstand hat. Der Artikel ist dem an italienische Unternehmen und deren juristische Berater adressierten Beiheft Guida al Diritto (Leitfaden des Rechts) der italienischen Wirtschaftszeitung Il Sole 24-Ore von Februar 2003 entnommen, das die kurz zuvor erfolgte Reform des italienischen Gesellschaftsrechts erläutert. Erläuterungen dieser Art sind unter bestimmten Umständen auch für bundesdeutsche Unternehmen und deren juristische Berater interessant, nämlich insbesondere dann, wenn die betreffenden Unternehmen an italienischen Unternehmen beteiligt oder von diesen abhängig sind. Die Übersetzung ist entsprechend zu dem Zweck anzufertigen, als Verständnishilfe für die besagte Zielgruppe zu dienen, und hat, wie das Original, eine informative Funktion. Entsprechend lauteten auch die Vorgaben an die Studierenden des Studiengangs ,Übersetzen ‘ am Fachbereich Übersetzen und Dolmetschen (Scuola Superiore di Lingue Moderne per Interpreti e Traduttori) der Universität Bologna in Forlì, die den Text als Prüfungstext im Rahmen des Kurses ,Fachübersetzung Italienisch-Deutsch` zu übersetzen hatten. Zum besseren Verständnis des thematisierten Sachverhalts wird im Folgenden der italienische Beispieltext zusammen mit einer Übersetzung in die bundesdeutsche Rechtssprache abgedruckt, die der oben beschriebenen Übersetzungssituation gerecht wird:

La costituzione della „nuova“ Srl e l'unipersonalità

Con la riforma del diritto societario trova conferma l'attuale assetto, che prevede la costituzione della società per:

- contratto stipulato da due o più soci;

- atto unilaterale dell'unico socio, fattispecie, come noto, già prevista dall'attuale ordinamento in seguito al recepimento della XII diret- 
tiva Cee di armonizzazione del diritto societario a opera del Dlgs 88/1993.

Peraltro, vengono ora introdotte alcune significative novità proprio in materia di responsabilità dell'unico socio di Srl; il previgente articolo 2497 del codice civile sanciva infatti che, in caso di insolvenza della società, per le obbligazioni sociali sorte nel periodo in cui le quote sono appartenute a un solo socio, questi risponde illimitatamente:

- quando sia una persona giuridica ovvero sia socio unico di altra società di capitali;

- quando non sia stato effettuato l'integrale versamento dei decimi, ai sensi dell'articolo 2476;

- fino a quando non sia stata attuata la pubblicità presso il registro delle imprese.

Le novità attengono:

1) alla scomparsa della previsione della responsabilità illimitata dell'unico socio che sia persona giuridica o dell'unico socio che sia unico socio di una pluralità di società di capitali; anche il socio persona giuridica di Srl o il socio unico di più società di capitali (anche per azioni, come la riforma ora consente) conserva il beneficio della limitata responsabilità, al pari del socio unico che sia persona fisica;

2) alla modificazione della disciplina inerente la responsabilità illimitata dell'unico socio.

Dispone, infatti, il nuovo articolo 2462 del codice civile che l'unico socio risponde illimitatamente, quindi oltre il valore dei suoi apporti al capitale sociale, qualora ricorrano le seguenti condizioni:

a) la società deve essere insolvente;

b) i conferimenti non siano stati interamente liberati (sia quelli dell'unico socio originario, sia quelli promessi ma non effettuati dai soci di società pluripersonale che, per qualsiasi motivo, sia divenuta unipersonale), e ciò tanto con riguardo ai conferimenti in sede di atto costitutivo quanto con riguardo a quelli conseguenti a operazioni sul capitale;

oppure, alternativamente,

c) non sia stata effettuata nel registro delle imprese la prescritta pubblicità dell'unicità del socio, attraverso il deposito della dichiarazione degli amministratori contenente l'indicazione del cognome e nome o della denominazione, della data e del luogo di nascita o di costituzione, del domicilio o della sede e cittadinanza dell’unico socio.

Precisa infine la norma che questa illimitata responsabilità non si estende al di là delle "obbligazioni sociali sorte nel periodo in cui l'in- 
tera partecipazione è appartenuta ad una sola persona”, e quindi non a quelle sorte in precedenza né a quelle originatesi successivamente.

Si nota quindi l'evidente cambiamento rispetto alla previgente normativa; se prima infatti si richiedeva la presenza contemporanea di entrambe le prescrizioni (vale a dire dell'integrale versamento dei decimi e della pubblicità presso il registro delle imprese) per continuare a godere del beneficio della limitata responsabilità pur se la società fosse insolvente, ora invece, con la disgiunzione "o" al posto della congiunzione previgente, è quindi sufficiente che almeno una delle due prescrizioni (versamento integrale o pubblicità) sussistano perché l'unico socio eviti il rischio dell'illimitata responsabilità in presenza di una situazione di insolvenza della società.

Übersetzung: Die Gründung der „neuen“ italienischen GmbH und die unbeschränkte Haftung des Alleingesellschafters

Auch nach der Reform des italienischen Gesellschaftsrechts wird die Srl gegründet durch:

- Vertrag zwischen zwei oder mehreren Gesellschaftern;

- einseitiges Rechtsgeschäft des Alleingesellschafters, was in der italienischen Rechtsordnung bekanntermaßen seit der Umsetzung der 12. EG-Richtlinie zur Harmonisierung des Gesellschaftsrechts durch die Gesetzesverordnung Nr. 88 von 1993 möglich ist.

Im Übrigen hat die Reform einige wichtige Neuerungen auf dem Gebiet der unbeschränkten Haftung des Alleingesellschafters der italienischen GmbH gebracht. So war in der alten Fassung von Artikel 2497 italienisches Zivilgesetzbuch festgelegt, dass der Alleingesellschafter im Fall der Zahlungsunfähigkeit der Gesellschaft für die Gesellschaftsverbindlichkeiten, die in dem Zeitraum entstanden sind, in dem die Geschäftsanteile einem einzigen Gesellschafter gehört haben, unter bestimmten Voraussetzungen unbeschränkt haftet, nämlich:

- wenn er eine juristische Person oder Alleingesellschafter einer anderen Kapitalgesellschaft ist;

- wenn die gemäß Artikel 2476 italienisches Zivilgesetzbuch zu leisenden Stammeinlagen nicht voll erbracht sind;

- solange die Publizitätspflicht nicht erfüllt, d.h. im Handelsregister nicht eingetragen worden ist, dass die Srl einen Alleingesellschafter hat und wer der Alleingesellschafter der $\mathrm{Srl}$ ist.

Die reformbedingten Neuerungen betreffen die folgenden Punkte:

1) Im Gegensatz zu früher ist die unbeschränkte Haftung des Alleingesellschafters der italienischen $\mathrm{GmbH}$ nicht mehr bereits dann gegeben, wenn es sich um eine juristische Person oder den Alleingesellschafter mehrerer Kapitalgesellschaften handelt. Wie der Alleingesellschafter, 
der eine natürliche Person ist, genießt vielmehr auch der Alleingesellschafter, der eine juristische Person oder Alleingesellschafter mehrerer Kapitalgesellschaften (infolge der gesellschaftsrechtlichen Reform auch von Aktiengesellschaften) ist, den Vorteil der beschränkten Haftung.

2) Darüber hinaus ändern sich die Voraussetzungen für die unbeschränkte Haftung des Alleingesellschafters der Srl.

Nach dem neuen Artikel 2462 italienisches Zivilgesetzbuch haftet der Alleingesellschafter nämlich unbeschränkt, d.h. über den Wert seiner Stammeinlagen hinaus, wenn die folgenden Bedingungen erfüllt sind:

a) die Gesellschaft ist zahlungsunfähig;

b) die bei der Gründung oder im Zuge einer effektiven Kapitalerhöhung zu leistenden Stammeinlagen (und zwar sowohl diejenigen des ursprünglichen Alleingesellschafters als auch die übernommenen, aber nicht geleisteten Stammeinlagen der Gesellschafter einer mit mehreren Personen betriebenen Gesellschaft, die, aus welchem Grund auch immer, zu einer Einpersonengesellschaft geworden ist) sind nicht voll erbracht worden;

oder aber

c) die für die italienische Einmann-GmbH vorgeschriebene Publizitätspflicht ist nicht erfüllt, d.h. es ist nicht die Erklärung der Geschäftsführer hinterlegt worden, aus der der Nachname und der Vorname oder die Firma des Alleingesellschafters hervorgeht sowie das Datum und der Ort seiner Geburt bzw. seiner Gründung, seine Anschrift oder sein Sitz sowie seine Staatsangehörigkeit.

Des Weiteren ist in der neuen Norm festgelegt, dass die unbeschränkte Haftung nur „Gesellschaftsverbindlichkeiten [betrifft], die in dem Zeitraum entstanden sind, in dem die Geschäftsanteile einem einzigen Gesellschafter gehört haben,“ und nicht auch in der vorausgehenden oder nachfolgenden Zeit.

Der offensichtliche Unterschied im Vergleich zu der vorherigen gesetzlichen Regelung ist der, dass die Bedingungen der vollen Erbringung der Stammeinlagen und der Erfüllung der Publizitätspflicht nicht mehr gemeinsam erfüllt sein müssen, damit der Alleingesellschafter im Fall der Zahlungsunfähigkeit der Gesellschaft sein Haftungsprivileg behält, sondern dass an die Stelle des „und“ nun ein „oder“ getreten ist und folglich nur eine der beiden Bedingungen (volle Erbringung der Stammeinlagen oder Erfüllung der Publizitätspflicht) erfüllt sein muss, damit der Alleingesellschafter im Fall der Zahlungsunfähigkeit der Gesellschaft nicht Gefahr läuft, unbeschränkt zu haften. 


\section{Das rechtliche Wissen des Rechtsübersetzers}

Das rechtliche Wissen des Rechtsübersetzers ist ein Teil der in Wiesmann (2004: 166-170) beschriebenen übersetzerischen Kompetenz, zu der traditionellerweise das Sprach-, das Kultur- und das Sach- bzw. Fachwissen zählt sowie die übersetzungsbezogene Kompetenz im weiteren Sinne, die darüber hinaus

1. das Wissen über die Anforderungen umfasst, die an eine Übersetzung zu einer bestimmten Zeit und in einer bestimmten Kultur sowie in der jeweiligen Übersetzungssituation gestellt werden,

2. das Wissen darüber, welches Wissen die Voraussetzung für die Anfertigung der Übersetzung ist, wie die eigenen Wissensbestände geordnet, verwaltet, aktualisiert und erweitert werden können, wo neues Wissen erworben und wie es erschlossen und in das bestehende Wissen eingegliedert werden kann, und letztlich

3. das Wissen, wie die Übersetzung im konkreten Fall auszuführen ist und welche Schritte die Ausführung der Übersetzung erfordert, sowie die Fähigkeit und Fertigkeit zu deren Anfertigung.

Was das Fachwissen Recht anbelangt, so betrifft das vom Rechtsübersetzer zur Übersetzung des Beispieltextes benötigte Wissen verschiedene Rechtsgebiete, nämlich in erster Linie das Gesellschaftsrecht und das ihm zugrunde liegende bürgerliche Recht mit dem Schuldrecht, des Weiteren das dem Gesellschaftsrecht übergeordnete Handelsrecht sowie - am Rande - das Insolvenzrecht. Abgesehen davon sind aber auch rechtstheoretische Kenntnisse erforderlich, insbesondere was die Normen und das System der Rechtsquellen betrifft.

Um den Text übersetzen zu können, muss der Rechtsübersetzer mit Bezug auf die italienische Rechtsordnung im Einzelnen wissen, zu welcher Gesellschaftsform die Srl gehört (zur Kapitalgesellschaft), wo sie gesetzlich geregelt ist (im Codice Civile, d.h. dem italienischen Zivilgesetzbuch, das neben dem bürgerlichen Recht u.a. das Handels- und das Gesellschaftsrecht sowie Normen des Insolvenzrechts umfasst), welches ihre Organe sind (neben den Gesellschaftern insbesondere die Geschäftsführer), dass sie mehrere, ggf. aber auch nur einen Gesellschafter haben kann und dass die Gesellschafter sowohl natürliche als auch juristische Personen sein können. Des Weiteren muss der Rechtsübersetzer wissen, wie eine $\mathrm{Srl}$ gegründet wird (durch Vertrag oder einseitiges Rechtsgeschäft), was die Kapitalaufbringung impliziert (die 
persönliche Verpflichtung der Gesellschafter zur vollen Erbringung der eigenen Stammeinlage), dass die Stammeinlagen grundsätzlich im Zusammenhang mit der Gründung der Srl, aber auch im Zusammenhang mit einer späteren Erhöhung des Stammkapitals gegen Einlagen (effektive im Gegensatz zur nominellen Kapitalerhöhung) zu leisten sind und in welchem Verhältnis einerseits Stammeinlage und Geschäftsanteil (nach der von jedem Gesellschafter zu leistenden Stammeinlage bestimmt sich sein Geschäftsanteil) und andererseits Stammkapital und Gesellschaftsvermögen (das aus den Stammeinlagen der Gesellschafter zusammengesetzte Stammkapital bildet den Haftungsfonds und den Grundstock des Gesellschaftsvermögens) zueinander stehen. Hinsichtlich des Haftungsrechts muss der Rechtsübersetzer über die Unterschiede zwischen dem alten und dem neuen Gesellschaftsrecht Bescheid wissen, da in dem zu übersetzenden Beispieltext gerade die gesetzlichen Neuerungen betreffend die unbeschränkte Haftung des Alleingesellschafters der Srl thematisiert werden. Diese ist im Fall der Zahlungsunfähigkeit der ansonsten grundsätzlich für die Gesellschaftsverbindlichkeiten mit dem Gesellschaftsvermögen haftenden Gesellschaft (beschränkte Haftung der Gesellschafter) nun unter veränderten Voraussetzungen gegeben, nämlich entweder wenn die Stammeinlagen nicht voll erbracht sind oder wenn die Publizitätspflicht nicht erfüllt ist. Außerdem ist sie nicht mehr an die Bedingung geknüpft, dass der Alleingesellschafter eine juristische Person oder Alleingesellschafter einer anderen Kapitalgesellschaft ist. In diesem Zusammenhang muss der Rechtsübersetzer gleichfalls wissen, dass das Handelsregister der Unterrichtung der Öffentlichkeit über die rechtserheblichen Verhältnisse von Unternehmen dient und dass im Handelsregister u.a. eingetragen werden muss (Publizitätspflicht), dass die Srl einen Alleingesellschafter hat und wer der Alleingesellschafter der Srl ist. Abgesehen davon muss der Rechtsübersetzer noch wissen, dass Normen, sofern sie aus einem Tatbestand und der angeordneten Rechtsfolge bestehen, Sollensbzw. Vorschriftscharakter haben, dass Gesetzesverordnungen in Italien den Gesetzen gleichstehen und dass das Recht der Staaten der Europäischen Gemeinschaft durch EG-Richtlinien angepasst, d.h. harmonisiert wird. In Bezug auf all diese Punkte muss der Rechtsübersetzer schließlich die Unterschiede zwischen dem italienischen und dem bundesdeutschen Recht kennen. 
Das rechtliche Wissen, das der Rechtsübersetzer zur Übersetzung des Beispieltextes benötigt, kann er zu einem gewissen Teil dem zu übersetzenden Text selbst entnehmen. Zu diesem Wissen gehört, a) dass die Gründung der Srl bereits nach dem alten Gesellschaftsrecht durch Vertrag oder einseitiges Rechtsgeschäft erfolgen konnte, b) dass die Voraussetzungen für die unbeschränkte Haftung des Alleingesellschafters (nicht volle Erbringung der Stammeinlagen und Nichterfüllung der $\mathrm{Pu}-$ blizitätspflicht) insofern geändert wurden, als die beiden Bedingungen nicht mehr gemeinsam erfüllt sein müssen, c) dass die ursprünglich vorgesehene dritte Voraussetzung (der Alleingesellschafter ist eine juristische Person oder Alleingesellschafter einer anderen Kapitalgesellschaft) entfällt, d) dass nicht nur natürliche, sondern auch juristische Personen Gesellschafter einer Srl sein können, e) dass eine $S r l$ als italienische Einmann-GmbH gegründet werden kann, aber auch zu einem späteren Zeitpunkt zu einer solchen werden kann, f) dass Stammeinlagen nicht nur im Zusammenhang mit der Gründung der $\mathrm{Srl}$ zu leisten sind, g) dass die Publizitätspflicht bei der italienischen Einmann-GmbH durch die Hinterlegung einer Erklärung mit den Angaben zum Alleingesellschafter erfüllt wird und $\mathrm{h}$ ) dass die unbeschränkte Haftung des Alleingesellschafters nur die Gesellschaftsverbindlichkeiten betrifft, die zu der Zeit entstanden sind, als die Gesellschaft eine Einpersonengesellschaft war.

Während das Thema im Text also ausführlich behandelt wird, da es für die angesprochene Zielgruppe der italienischen Unternehmen und deren juristische Berater neu ist, wird das übrige rechtliche Wissen als Vorwissen vorausgesetzt. Für den nicht juristisch geschulten Rechtsübersetzer, der über dieses Vorwissen nicht verfügt, heißt das, dass er sich das betreffende Wissen aus übersetzerischen Hilfsmitteln aneignen muss. Wie sich zeigen wird, ist dies jedoch nicht ganz unproblematisch.

\section{Die Hilfsmittel des Rechtsübersetzers}

Aus welchen Typen von übersetzerischen Hilfsmitteln kann der Rechtsübersetzer nun das rechtliche Wissen beziehen, das er nicht hat und das er auch dem zu übersetzenden Text nicht entnehmen kann? Von den in der Hilfsmitteltypologie von Nord (2002: 158-163) genannten Hilfsmitteltypen kommen grundsätzlich Hilfstexte wie Hintergrundtexte und 
Paralleltexte sowie Nachschlagewerke in Betracht, zu denen u.a. lexikographische Werke und benutzerdefinierte Verzeichnisse gezählt werden. Während der Unterschied zwischen Hintergrund- und Paralleltexten darin liegt, dass in Ersteren die gleichen Sachverhalte thematisiert werden wie in dem zu übersetzenden Text und dass Letzteren nicht nur Sach- bzw. Fach- sondern auch sprachliches Wissen zu entnehmen ist, da sie zur gleichen Textsorte wie der zu übersetzende Text gehören und in der Sprache abgefasst sind, in die übersetzt wird, ist der Unterschied zwischen den lexikographischen Werken und den benutzerdefinierten Verzeichnissen darin zu sehen, dass die Zweitgenannten von dem und für den Benutzer selbst geschaffen werden und die unterschiedlichsten Formen annehmen können, während die Erstgenannten von Lexikographen geschaffen werden und an bestimmte Benutzergruppen adressiert sind. Handelt es sich bei den lexikographischen Werken um fachbezogene Werke (siehe Wiesmann 2004: 184), so wird fachliches Wissen v.a. in Fachenzyklopädien und fachlichen Allbüchern vermittelt, für die kennzeichnend ist, dass sie im ersten Fall primär Informationen zu fachlichen Gegenständen und im zweiten Fall Informationen zu fachlichen und zu fachsprachlichen Gegenständen liefern. Alle übersetzerischen Hilfsmittel können dabei grundsätzlich in gedruckter oder in elektronischer Form vorliegen, was insbesondere bei den lexikographischen Werken heißt, dass sie Datenbanken sein können.

Für die Übersetzung des italienischen Beispieltextes in die bundesdeutsche Rechtssprache kommen konkret die folgenden Hilfsmittel in Frage:

1. lexikographische Werke

- Rechtsenyzklopädien: v.a. Galgano 1996, Bianca 2001, De Stefanis 2005, Enciclopedia Giuffrè 1958-2004, Garzantina Diritto 2001, Dizionario Giuridico Simone 2001, Creifelds 2007, DRL 2001, DRL 2003;

- rechtliche Allbücher: Ciola 2000, Maganzi 2001, Bullo 2003;

- Datenbanken: BISTRO, JUSLEX, TERMIT.

2. Hintergrundtexte

- Gesetzestexte: Codice Civile, BGB, HGB, GmbHG;

- juristische Lehrbücher: v.a. Ferrara/Corsi 2006, Hueck/Windbichler 2003, Musielak 2007, Galgano 2004, Kindler 2007, Kindler 2002.

Während sich als Paralleltexte für die Rechtsübersetzung im Allgemeinen Formularbücher eignen, da sie Textmuster für die im jewei- 
ligen Zusammenhang interessierenden Textsorten enthalten, erfüllen für die Übersetzung des Beispieltextes angesichts seiner Einordnung als Text der Rechtslehre (siehe Wiesmann 2004: 60-66) die zu den Hintergrundtexten zu zählenden, Einführungen ins Recht oder in einzelne Rechtsgebiete liefernden juristischen Lehrbücher die Funktion von Paralleltexten, weshalb Paralleltexte nicht als eigener Hilfsmitteltyp berücksichtigt werden. Nicht berücksichtigt werden schließlich auch die benutzerdefinierten Verzeichnisse, da sie in aller Regel nur dem Benutzer selbst zugänglich sind.

\section{Problematik der übersetzerischen Hilfsmittel}

Was die Problematik der für die Übersetzung des Beispieltextes zur Verfügung stehenden Hilfsmittel des Rechtsübersetzers anbelangt, so wird sie allein mit Blick auf das vom Rechtsübersetzer benötigte rechtliche Wissen behandelt, das für das Verstehen und die Übersetzung der nicht wörtlich übersetzbaren Textstellen besondere Relevanz erlangt. Rechtliche Informationen werden v.a. von Rechtsenzyklopädien, juristischen Lehrbüchern und Gesetzestexten geliefert, finden sich aber auch in rechtlichen Allbüchern und Datenbanken, wobei Rechtsübersetzer als Zielgruppe in recht unterschiedlichem Maße berücksichtigt werden. Während Rechtsübersetzer als Adressaten von juristischen Lehrbüchern keine Berücksichtigung finden, kann bei Gesetzestexten, die sich v.a. an die Juristen, aber auch an die Bürger des betreffenden Staates richten, allenfalls von einer indirekten Berücksichtigung die Rede sein. Anders verhält es sich bei den rechtlichen Allbüchern und den Datenbanken, die - mit Ausnahme der allein für den Rechtsübersetzer entworfenen und entwickelten Datenbank JUSLEX - explizit sowohl für Juristen als auch für Rechtsübersetzer gedacht sind, wohingegen bei Rechtsenzyklopädien Rechtsübersetzer wiederum nur implizit als interessierte Nichtjuristen mitberücksichtigt sind.

Bei der näheren Betrachtung der betreffenden Hilfsmittel ist zunächst festzustellen, dass es hinsichtlich der Qualität von juristischen Lehrbüchern und Gesetzestexten zwischen den auf das italienische und den auf das bundesdeutsche Recht bezogenen Hilfsmitteln keine nennenswerten Unterschiede gibt, ebenso wie die rechtlichen Allbücher und die Datenbanken beide Rechtsordnungen gleich gut behandeln. Anders verhält es sich dagegen bei den Rechtsenzyklopädien. Dort stehen einem 
umfassend über das bundesdeutsche Recht Auskunft gebenden, informativen und aktuellen DRL 2001 in drei Bänden, zu dem es den Ergänzungsband DRL 2003 gibt, und einem knapperen einbändigen, entsprechend weniger Informationen über das bundesdeutsche Recht liefernden, dafür aber noch aktuelleren und dank der elektronischen Form auch leichter zugänglichen Creifelds 2007, im italienischen Recht eine Vielzahl von vorwiegend gedruckt vorliegenden Rechtsenzyklopädien gegenüber, die sich in Umfang, Aktualität und Qualität sehr stark voneinander unterscheiden. Die umfassendsten rechtlichen Informationen zu den aufgenommenen Stichwörtern liefert die 46-bändige Enciclopedia Giuffrè 1958-2004, die zwar zu den besten italienischen Rechtsenzyklopädien zählt, aber den Nachteil hat, dass ihr erster Band von 1958 stammt. Ebenfalls sehr gut, aber auch verhältnismäßig alt ist Galgano 1996 in zwei Bänden, während die einbändige Rechtsenzyklopädie Bianca 2001 zwar aktuell ist, aber leider nur das italienische bürgerliche Recht betrifft. Auf das italienische Gesellschaftsrecht bezogen ist hingegen De Stefanis 2005, eine einbändige Rechtsenzyklopädie, die abgesehen von der alphabetischen Anordnung der Stichwörter eher einer Einführung in die Reform des italienischen Gesellschaftsrechts gleicht, in der die gesetzlichen Neuerungen erläutert, nicht aber die als bekannt vorausgesetzten gesellschaftsrechtlichen Termini definiert werden. Nach der einbändigen Garzantina Diritto 2001 bildet die gleichfalls einbändige, nicht nur in gedruckter Form und als CD-ROM, sondern auch frei im Internet verfügbare (http://www.simone.it/cgi-local/ Dizionari/newdiz.cgi ?index,5,A) Rechtsenzyklopädie Dizionario Giuridico Simone 2001 qualitätsmäßig das Schlusslicht.

Angesichts dieser Ausgangslage interessiert besonders, welchen Beitrag die a) an Juristen, b) an Juristen und Nichtjuristen bzw. c) an Juristen und Rechtsübersetzer adressierten übersetzerischen Hilfsmittel v.a. zum Verstehen und zur Übersetzung nicht wörtlich übersetzbarer, rechtliches Wissen in besonderem Maße voraussetzender Textstellen leisten, inwieweit der Umgang mit ihnen problematisch ist und welche Konsequenzen daraus für den Entwurf und die Entwicklung von JUSLEX gezogen wurden.

Bei Rechtsenyzklopädien und Gesetzestexten ist das erste Problem bereits die typographische Gestaltung. Die vielzähligen rechtlichen Informationen finden auf engem Raum Platz, sind häufig zweispaltig angeordnet und typographisch nur geringfügig voneinander abgesetzt. 
Es verwundert daher nicht, dass für den Rechtsübersetzer, der sich mit neuen Sachverhalten auseinandersetzen muss, der erste Blick in diese Hilfsmittel demotivierend ist. Dazu kommt erschwerend der Abstraktionsgrad der Formulierungen hinzu, der wiederum in Rechtsenyzklopädien und Gesetzestexten nicht selten ist. Schlägt der Rechtsübersetzer beispielsweise unter fattispecie in der Garzantina Diritto 2001 nach, so sieht er sich mit abstrakten Formulierungen wie der folgenden konfrontiert:

(1) fattispecie [...] termine con il quale si indica una situazione-tipo, considerata astrattamente da una norma come produttiva di effetti giuridici (fattispecie legale o astratta). [...]

Übersetzung: Tatbestand [...] Terminus, mit dem auf einen typischen, in der Norm abstrakt erfassten Sachverhalt Bezug genommen wird, mit dem eine Rechtsfolge verbunden ist (gesetzlicher Tatbestand). [...]

Solche Formulierungen können u.U. - und so auch im Beispielfall dazu führen, dass der Rechtsübersetzer nicht in der Lage ist zu beurteilen, ob der Terminus im Text in der im konsultierten Hilfsmittel definierten Weise verwendet wird. Anschaulicher sind dagegen i.d.R. die juristischen Lehrbücher, v.a. wenn sie die juristische Arbeitsmethode erklären oder anhand von Fällen verdeutlichen. So wird beispielsweise in Musielak 2007 der Terminus Tatbestand erst im Anschluss an eine ausführliche Beschreibung der Methode der Rechtsanwendung definiert.

Eines der größten Probleme, das der Rechtsübersetzer mit allen Hilfsmitteln hat, die nicht ausschließlich für ihn entworfen und entwickelt worden sind, sondern Juristen ansprechen oder zumindest mitansprechen, ist jedoch, dass er die Termini, die er sucht, dort u.U. nur über größere Umwege findet. So sind Termini wie apporti (Beiträge) oder decimi (Zehntel) für Juristen nämlich insofern uninteressant, als der fachlichere, der Gebrauchsnorm des Gesetzgebers entsprechende Terminus für apporti conferimenti (Einlagen) ist, und der Terminus deci$m i$ auf einen Juristen hinlänglich bekannten Sachverhalt verweist, nämlich den, dass nach dem alten italienischen Gesellschaftsrecht bei der Gründung der $\mathrm{Srl}$ eine bestimmte Zahl von Zehnteln der Stammeinlagen (drei Zehntel oder zehn Zehntel, je nachdem) zu leisten waren. Da in den an Juristen adressierten oder mitadressierten Hilfsmitteln die Be- 
griffe und nicht deren unterschiedliche Benennungen im Vordergrund stehen, finden Termini der genannten Art selten oder gar nicht als Stichwörter in lexikographische Werke Eingang bzw. sind selten oder gar nicht im Register von Hintergrundtexten verzeichnet. Dem Rechtsübersetzer bleibt daher im besten Fall die Möglichkeit der elektronischen Volltextsuche, im schlimmsten Fall die Lektüre mehr oder weniger umfassender Einträge aus lexikographischen Werken bzw. mehr oder weniger langer Kapitel aus Hintergrundtexten. Und Letzteres setzt voraus, dass von übergeordneten Zusammenhängen ausgegangen und Verweisen nachgegangen wird, was entsprechend zeitaufwendig ist. Im Fall des Terminus apporti, der im Satz

(2) Dispone, infatti, il nuovo articolo 2462 del codice civile che l'unico socio risponde illimitatamente, quindi oltre il valore dei suoi apporti al capitale sociale, qualora ricorrano le seguenti condizioni: [...]

Übersetzung: Nach dem neuen Artikel 2462 italienisches Zivilgesetzbuch haftet der Alleingesellschafter nämlich unbeschränkt, d.h. über den Wert seiner Stammeinlagen hinaus, wenn die folgenden Bedingungen erfüllt sind: [...]

vorkommt, wäre beispielsweise die Konsultation des auf den Eintrag conferimenti verweisenden Eintrags capitale sociale in der Rechtsenzyklopädie Garzantina Diritto 2001 weiterführend gewesen, da dies dem Rechtsübersetzer geholfen hätte zu verstehen, dass apporti ein Synonym zum Terminus conferimenti ist, für den in einem rechtlichen Allbuch wie Ciola 2000 und in einer Datenbank wie BISTRO dann mit Bezug auf die Gesellschaft mit beschränkter Haftung die Übersetzung ,Stammeinlagen` angeführt wird.

Ein weiteres großes Problem, das der Rechtsübersetzer mit den an Juristen adressierten oder mitadressierten Hilfsmitteln hat, ist die u.U. nicht restlose Beantwortung seiner Fragen. So findet sich zum Terminus decimi im Satz

(3) il previgente articolo 2497 del codice civile sanciva infatti che, in caso di insolvenza della società, per le obbligazioni sociali sorte nel periodo in cui le quote sono appartenute a un solo socio, questi risponde illimitatamente: [...] quando non sia stato effettuato l'integrale versamento dei decimi, ai sensi dell'articolo 2476 [...].

Übersetzung: So war in der alten Fassung von Artikel 2497 italienisches Zivilgesetzbuch festgelegt, dass der Alleingesellschafter im Fall der Zahlungsunfähigkeit der Gesellschaft für die Gesellschafts- 
verbindlichkeiten, die in dem Zeitraum entstanden sind, in dem die Geschäftsanteile einem einzigen Gesellschafter gehört haben, unter bestimmten Voraussetzungen unbeschränkt haftet, nämlich: [...] wenn die gemäß Artikel 2476 italienisches Zivilgesetzbuch zu leistenden Stammeinlagen nicht voll erbracht sind [...].

zwar ein Eintrag im Dizionario Giuridico Simone 2001, doch ist dieser in der betreffenden Rechtsenzyklopädie lediglich auf die italienische Aktiengesellschaft (SpA, d.h. società per azioni) bezogen. Und beim Blick in die alte Fassung von Artikel 2476 italienisches Zivilgesetzbuch tauchen neue Fragen auf, da einerseits davon die Rede ist, dass die Geldeinlagen bei Gründung der Gesellschaft durch einseitiges Rechtsgeschäft ,voll` zu leisten sind, andererseits auf die alte Fassung von Artikel 2329, Nr. 2 italienisches Zivilgesetzbuch verwiesen wird, nach dem ,mindestens drei Zehntel“ der Geldeinlagen bei einem Kreditinstitut einzuzahlen sind.

Findet der Rechtsübersetzer in den von ihm konsultieren Hilfsmitteln hingegen Erklärungen zu den ihm unbekannten Termini, so liegt ein Problem häufig darin, dass die Erklärungen weitere ihm unbekannte Termini enthalten, die er ihrerseits nachschlagen muss. Auch verlangt die Komplexität der Darstellung - wie das Beispiel Geschäftsanteil zeigt - häufig die Konsultation mehrerer Hilfsmittel zu ein und demselben Problem. Nach der Rechtsenzyklopädie Creifelds 2007 ist Geschäftsanteil

(4) der Anteil des Gesellschafters am $\rightarrow$ Gesellschaftsvermögen. Er stellt den Anteil an einer $\rightarrow$ Gesamthandsgemeinschaft dar, ist vom $\rightarrow$ Kapitalanteil zu unterscheiden und wird nicht in einer Geldsumme, sondern durch einen bestimmten Bruchteil ausgedrückt. Der Gesellschaftsanteil kann beim Ausscheiden eines Gesellschafters auf die anderen Gesellschafter durch $\rightarrow$ Anwachsung übergehen; an seine Stelle tritt dann das $\rightarrow$ Abfindungsguthaben. Der G. kann nur dann übertragen werden (vgl. § 719 BGB), wenn alle Gesellschafter zustimmen oder die Übertragung im Gesellschaftsvertrag zugelassen ist. Der G. kann gepfändet werden ( $\rightarrow$ Pfändung, § 859 I ZPO); der Pfändungsgläubiger ist dann berechtigt, die Gesellschaft zu kündigen. Bei einer $\rightarrow$ Gesellschaft mit beschränkter Haftung bestimmt sich der G. nach dem Betrag der übernommenen $\rightarrow$ Stammeinlage in ihrem Verhältnis zum $\rightarrow$ Stammkapital ( $\$ 14$ GmbHG). Er ist veräußerlich und vererblich (§ 15 I GmbHG). In der Satzung kann bestimmt sein, dass die Veräußerung des G. der Zustimmung der GmbH bedarf (sog. vinkulierter $G$.). Die Abtretung des G. und die Verpflichtung hierzu ist nur 
wirksam, wenn sie notariell beurkundet wird (§ 15 III, IV GmbHG). Jeder Gesellschafter kann weitere G.e hinzuerwerben; doch behalten diese ihre Selbständigkeit (§ 15 II GmbHG). Wenn die Stammeinlage verzögert eingezahlt wird, kann der G. unter den Voraussetzungen des $\S 21$ GmbHG zugunsten der Gesellschaft kaduziert ( $\rightarrow$ Kaduzierung) oder öffentlich versteigert werden (§ $23 \mathrm{GmbHG}$ ). Durch $\rightarrow$ Amortisation (Einziehung) kann ein G. erlöschen. [...].

nach der Rechtsenzyklopädie DRL 2001 hingegen bezeichnet der Geschäftsanteil

(5) den Inbegriff der Rechtsstellung, d.h. der Mitgliedschaftsrechte und -pflichten, eines GmbH-Gesellschafters. Er drückt die Gesellschafterbeteiligung an der GmbH aus. Der Geschäftsanteil ist mit der $\rightarrow$ Stammeinlage insofern verknüpft, als ihm ein Nennbetrag in Höhe der Stammeinlage zugeschrieben wird. Danach richtet sich das Beteiligungsverhältnis, und nach diesem wiederum bemessen sich die wichtigsten Rechte und Pflichten (z.B. das Stimmrecht). Allerdings kann ein Geschäftsanteil auch mit Sonderrechten ausgestattet oder mit Sonderpflichten belastet sein. Der wirtschaftliche Wert des Geschäftsanteils richtet sich nach dem Wert des Gesellschaftsvermögens. [...]

Das macht es für den Rechtsübersetzer entsprechend schwer zu beurteilen, ob italienische und bundesdeutsche Begriffe einander entsprechen. Diesbezüglich helfen ihm am besten rechtliche Allbücher und Datenbanken, die eine Brücke von einer Rechtsordnung zur anderen schlagen und über die Entsprechung von Begriffen unterschiedlicher Rechtsordnungen Auskunft geben. Sollen mit den betreffenden Hilfsmitteln allerdings Juristen und Rechtsübersetzer gleichermaßen angesprochen werden, dann wird davon ausgegangen, dass mit einer Begriffsorientierung deren Bedürfnissen in der gleichen Weise Rechnung getragen werden kann. Das führt dann auch in diesen Hilfsmitteln dazu, dass unterschiedliche Benennungen (apporti und conferimenti) ein und desselben Begriffs nicht systematisch verzeichnet werden und dass Benennungen nicht den Ausgangspunkt für die Beschreibung von Begriffen darstellen. Ein Beispiel hierfür ist der Terminus pubblicità, der im Beispieltext u.a. an der folgenden, für das Verstehen und die Übersetzung nicht unproblematischen Stelle vorkommt:

(6) il previgente articolo 2497 del codice civile sanciva infatti che, in caso di insolvenza della società, per le obbligazioni sociali sorte nel periodo in cui le quote sono appartenute a un solo socio, questi risponde illimi- 
tatamente $[. .$.$] fino a quando non sia stata attuata la pubblicità presso$ il registro delle imprese.

Übersetzung: So war in der alten Fassung von Artikel 2497 italienisches Zivilgesetzbuch festgelegt, dass der Alleingesellschafter im Fall der Zahlungsunfähigkeit der Gesellschaft für die Gesellschaftsverbindlichkeiten, die in dem Zeitraum entstanden sind, in dem die Geschäftsanteile einem einzigen Gesellschafter gehört haben, unter bestimmten Voraussetzungen unbeschränkt haftet, nämlich [...] solange die Publizitätspflicht nicht erfüllt, d.h. im Handelsregister nicht eingetragen worden ist, dass die Srl einen Alleingesellschafter hat und wer der Alleingesellschafter der Srl ist.

Während das rechtliche Allbuch Ciola 2000 lediglich pubblicità del bilancio verzeichnet und weder in der Rechtsenzyklopädie De Stefanis 2005 noch in dem juristischen Lehrbuch Ferrara/Corsi 2006 irgendein Eintrag zu pubblicità, geschweige denn zu pubblicità presso il registro delle imprese vorhanden ist, wird in der Garzantina Diritto 2001 neben pubblicità in der Bedeutung, Werbung“ zwar pubblicità degli atti giuridici angeführt, doch kann der Rechtsübersetzer, der nicht weiß, dass darunter auch die rechtserheblichen Verhältnisse von Unternehmen fallen, damit kaum etwas anfangen. Anders wäre es gewesen, wenn in den von ihm konsultierten Hilfsmitteln zunächst pubblicità als ,Zugänglichkeit von Informationen für bestimmte Informationsberechtigte‘ definiert und dann angegeben worden wäre, in welchen Zusammenhängen (nämlich im Zusammenhang mit den rechtserheblichen Verhältnissen von Unternehmen ebenso wie im Zusammenhang mit den Eigentumsverhältnissen von Personen und den persönlichen Grundverhältnissen des Menschen) sie relevant ist. Abgesehen davon stellt sich in Bezug auf pubblicità presso il registro delle imprese das Problem, dass die Unmöglichkeit der wörtlichen Übersetzung mit ,Publizität im Handelsregister' genauere Angaben darüber erfordert, in was die Publizitätspflicht besteht, von der in der besagten Textstelle die Rede ist. Hier hilft am besten die alte Fassung des italienischen Zivilgesetzbuches weiter, über dessen Registereintrag pubblicità nelle società a responsabilità limitata auf Artikel 2475 bis verwiesen wird, aus dem hervorgeht, dass auch nach dem alten Gesellschaftsrecht im Handelsregister einzutragen war, dass die Srl einen Alleingesellschafter hat und wer der Alleingesellschafter der $\mathrm{Srl}$ ist. 
Ein weiteres Problem ist, dass der juristische Sprachgebrauch in den Hilfsmitteln des Rechtsübersetzers keine Berücksichtigung findet. Aus Gründen der Sprachökonomie werden auch in juristischen Texten Termini abkürzend verwendet. So stehen im Beispieltext - ohne dass sich darauf in den an Juristen adressierten oder mitadressierten Hilfsmitteln Hinweise fänden - denominazione für denominazione sociale und quote für quote sociali, was bedeutet, das der erstgenannte Terminus nicht mit ,Bezeichnung', sondern mit ,Firma‘ übersetzt werden muss, und der zweitgenannte nicht mit ,Anteile‘, sondern mit ,Geschäftsanteile‘. Auf den Sprachgebrauch zurückzuführen sind auch die Probleme mit apporti und decimi, auf die bereits eingegangen worden ist, sowie das Problem mit recepimento della XII direttiva Cee (Umsetzung der 12. EG-Richtlinie), das der Rechtsübersetzer erst über Umwege lösen kann, die ihn erkennen lassen, dass recepimento - oder besser: ricezione - im Zusammenhang mit EG-Richtlinien gleich Umsetzung ist. Auch von den operazioni sul capitale - wörtlich ,kapitalbeeinflussende Geschäfte` - in

(7) Dispone, infatti, il nuovo articolo 2462 del codice civile che l'unico socio risponde illimitatamente, quindi oltre il valore dei suoi apporti al capitale sociale, qualora ricorrano le seguenti condizioni: [...] i conferimenti non siano stati interamente liberati (sia quelli dell'unico socio originario, sia quelli promessi ma non effettuati dai soci di società pluripersonale che, per qualsiasi motivo, sia divenuta unipersonale), e ciò tanto con riguardo ai conferimenti in sede di atto costitutivo quanto con riguardo a quelli conseguenti a operazioni sul capitale [...].

Übersetzung: Nach dem neuen Artikel 2462 italienisches Zivilgesetzbuch haftet der Alleingesellschafter nämlich unbeschränkt, d.h. über den Wert seiner Stammeinlagen hinaus, wenn die folgenden Bedingungen erfüllt sind: [...] die bei der Gründung oder im Zuge einer effektiven Kapitalerhöhung zu leistenden Stammeinlagen (und zwar sowohl diejenigen des ursprünglichen Alleingesellschafters als auch die übernommenen, aber nicht geleisteten Stammeinlagen der Gesellschafter einer mit mehreren Personen betriebenen Gesellschaft, die, aus welchem Grund auch immer, zu einer Einpersonengesellschaft geworden ist) sind nicht voll erbracht worden [...].

führt kein direkter Weg zur effektiven Kapitalerhöhung, aber immerhin lässt der Vergleich zwischen der Textstelle im Beispieltext, der italienischen Rechtsenzyklopädie De Stefanis 2005 und der deutschen Rechtsenzyklopädie DRL 2001 den Schluss zu, dass keine Kapitalhe- 
rabsetzung gemeint sein kann, sondern nur eine bestimmte Form von Kapitalerhöhung, nämlich die Kapitalerhöhung gegen Einlagen oder effektive Kapitalerhöhung. Ähnlich verhält es sich in der gleichen Textstelle mit conferimenti promessi und conferimenti liberati - wörtlich ,versprochene Stammeinlagen` und ,befreite Stammeinlagen' -, mit denen im ersten Fall auf die Verpflichtung zur Erbringung der Stammeinlagen - im bundesdeutschen Recht Übernahme genannt - und im zweiten Fall auf die Erfüllung der Verpflichtung - im bundesdeutschen Recht Erbringung der Stammeinlagen (bei Geldeinlagen auch Einzahlung) genannt - Bezug genommen wird. Mangels expliziter Erklärungen in den an Juristen adressierten oder mitadressierten Hilfsmitteln müssen die Bedeutungen der Termini und die dahinterstehenden Begriffe also vom Rechtsübersetzer oft erschlossen werden.

Abschließend kann festgehalten werden, dass die Wissensaufbereitung dem Rechtsübersetzer in allen Hilfsmitteln, die an Juristen adressiert oder mitadressiert sind, Schwierigkeiten bereitet und seinen Bedürfnissen nicht oder zumindest nicht umfassend gerecht wird. Zwar müssen Gesetze trotz ihrer Abstraktheit und trotz der Komplexität, mit der dort die Sachverhalte dargestellt werden, vom Rechtsübersetzer insofern konsultiert werden, als juristische Texte kaum ohne Bezüge auf Gesetze auskommen und Gesetze folglich als komplementär zu juristischen Texten zu betrachten sind, bei den anderen Hilfsmitteln bedarf es jedoch einer Alternative. Eine solche Alternative will die allein für den Rechtsübersetzer entworfene und entwickelte Datenbank JUSLEX sein.

\section{JUSLEX - die Datenbank für den Rechtsübersetzer}

Der Entwurf und die Entwicklung von JUSLEX stützen sich einerseits auf übersetzungspraktische Erfahrungen im Umgang mit den Hilfsmitteln des Rechtsübersetzers, andererseits werden die daraus abgeleiteten Erkenntnisse mit übersetzungs- und sprachtheoretischen, metafachlexikographischen und terminologiewissenschaftlichen Überlegungen zusammengeführt und mit den einschlägigen Ergebnissen der Wörterbuchforschung, der Hilfsmittelbenutzungsforschung und der Evaluierung von elektronischen Wörterbüchern und Terminologieverwaltungssystemen in Verbindung gebracht (siehe Wiesmann 2004). Als Datenbank für den Rechtsübersetzer geht es JUSLEX darum, den sich mit 
den Bedürfnissen von Juristen nur teilweise deckenden Bedürfnissen von Rechtsübersetzern gerecht zu werden. Dies wird v.a. durch die folgenden Merkmale der Datenbank gewährleistet:

- In JUSLEX wird sowohl die rechtssprachliche Terminologie als auch die rechtssprachliche Phraseologie verwaltet. Folglich findet der Rechtsübersetzer dort nicht nur zu conferimenti, sondern auch zu conferimenti liberati einen eigenen Eintrag mit dem nach dem Eintragsmodell vorgesehenen Angaben.

- Zur rechtssprachlichen Terminologie werden in JUSLEX alle von Juristen in einer rechtssprachlichen Bedeutung verwendeten Termini gezählt, auch die weniger fachlichen, der Gebrauchsnorm des Gesetzgebers nicht entsprechenden Termini wie apporti und die abkürzend verwendeten Termini wie denominazione.

- Die rechtssprachliche Terminologie und Phraseologie wird in JUSLEX rezeptions- und produktionsorientiert verwaltet, d.h. so, dass die Datenbank für die Übersetzung in beide Richtungen, aber auch übersetzungsunabhängig zum Erwerb von rechtlichem Wissen einsetzbar ist.

- JUSLEX ist als enzyklopädisches Rechtswörterbuch oder, m.a.W., als rechtliches Allbuch mit Angaben zu den Rechtsordnungen (Italien, Deutschland, Österreich, EU, usw.) konzipiert, denen die rechtssprachlichen Einheiten zugehören, sowie zu den (annähernd, teil- oder nulläquivalenten) Beziehungen, die zwischen ihnen bestehen.

- Jede terminologische oder phraseologische Einheit ist nach dem Eintragsmodell mit den folgenden Angaben versehen: a) einer grammatikalischen Angabe zur terminologischen oder phraseologischen Einheit, b) der Angabe der Rechtsordnung und der Rechtssprache (da es für den Rechtsübersetzer einen großen Unterschied macht, ob er aus der italienischen in die bundesdeutsche oder die österreichische Rechtssprache übersetzt), c) der Rechtsgebietsangabe, d) einer Definition mit Quellenangabe (die, sofern sich in den Hilfsmitteln des Rechtsübersetzers keine oder keine geeignete Definition findet, selbst erarbeitet und so verständlich wie möglich formuliert wird), e) Informationen zur Vertiefung, d.h. weiterführende Informationen zum Recht (die über die Definition hinaus Begriffe klären, sie in größere, übersichtlich dargebotene rechtliche Zusam- 
menhänge stellen und sie, falls erforderlich, veranschaulichen), f) einer Kontextangabe, g) einer Phraseologieangabe, h) Verweisen auf andere Einträge, i) Anmerkungen und Quellenangaben zur terminologischen oder phraseologischen Einheit sowie zu deren Synonymen bzw. Varianten.

- $\mathrm{Zu}$ jedem ausgangssprachlichen Eintrag wird ein darauf bezogener zielsprachlicher Eintrag erstellt, der die folgenden Angaben vorsieht: a) eine grammatikalische Angabe zur Übersetzung, b) die Angabe der Rechtsordnung und der Rechtssprache, c) die Angabe des Äquivalenzgrades bzw. der Kennzeichnung, dass es sich um einen Übersetzungsvorschlag handelt, d) Anmerkungen und Quellenangaben. Zu registro delle imprese ist entsprechend angegeben, dass es sich um ein Teiläquivalent zum bundesdeutschen Begriff Handelsregister handelt und dass auch eine Übersetzung mit dem der bundesdeutschen Rechtsordnung unbekannten ,Unternehmensregister` möglich ist.

- Die Eintragsstrukturen für Ausgangs- und Zielsprache sind in JUSLEX je unterschiedliche, damit zu jedem übersetzungsunabhängig erstellten ausgangssprachlichen Eintrag (z.B. zu fattispecie) genau der dazu passende zielsprachliche Eintrag (Tatbestand) geliefert werden kann. Durch die Möglichkeit des Wechsels der Sprachrichtung wird jedoch gewährleistet, dass der Benutzer zu jedem zielsprachlichen Eintrag genau diejenigen Angaben konsultieren kann, die zu einem ausgangssprachlichen Eintrag vorgesehen sind.

- Um (wie bei registro delle imprese und Handelsregister) die Darstellung der Inkongruenzen zu ermöglichen, die sich aus der Bindung der Rechtssprachen an die Rechtsordnungen ergeben, um (wie im Fall von Haftung) Bedeutungen differenzieren zu können (Haftung ist nicht nur ,die Verpflichtung des Schuldners, gegenüber dem Gläubiger für eine aus einem Schuldverhältnis herrührende Schuld mit seinem Vermögen einzustehen', sondern auch ,die Verpflichtung, für andere einzustehen' und ,die durch gesetzliche Bestimmungen begründete Schuld einer anderen Person gegenüber') und um dem Rechtsübersetzer entgegenzukommen, der im Text grundsätzlich zunächst mit Benennungen konfrontiert wird, ist das Eintragsmodell insofern ein benennungsorientiertes, also von den Benennungen ausgegangen wird, um zu den Begriffen zu gelangen. 
Durch die gemeinsame Verwaltung von Synonymen und Varianten wird dann jedoch in jedem Fall eine begriffsorientierte Sicht ermöglicht. Entsprechend werden die Termini conferimenti und apporti sowohl in einem Eintrag conferimenti mit dem Synonym apporti als auch in einem Eintrag apporti mit dem Synonym conferimenti erfasst.

- Schließlich wird in JUSLEX eine umfassende Datenvernetzung gewährleistet, und zwar über ein semiotisch durch die Einbindung von Graphiken erweitertes und durch die Verlinkung mit Korpustexten (v.a. Formularbücher und Gesetze) aus der Datenbank herausführendes Hypertextsystem aus impliziten und expliziten Verweisen und Links zwischen den terminologischen und den phraseologischen Einheiten, ihren Synonymen und Varianten sowie ihren Übersetzungen.

\section{Bibliographie}

\section{Übersetzerische Hilfsmittel (außer Datenbanken und Gesetzestexten)}

Bianca 2001 = Bianca, Cesare M. et al. 2001: Lessico di diritto civile. Milano: Giuffrè.

Bullo 2003 = Bullo, Francesca et al. 2003: Terminologisches Wörterbuch zum Vertragsrecht italienisch / deutsch. Dizionario terminologico del diritto dei contratti italiano / tedesco. München: Beck.

Ciola 2000 = Ciola, Bruno et al. 2000: Terminologisches Wörterbuch zum Gesellschaftsrecht italienisch / deutsch. Dizionario terminologico del diritto societario italiano / tedesco. München: Beck.

Creifelds 2007 = Weber, Klaus (ed.) 2007: Creifelds Rechtswörterbuch. Buch und CDROM. München: Beck.

De Stefanis 2005 = De Stefanis, Cinzia 2005: Dizionario delle nuove società di capitali. Piacenza: La Tribuna.

Dizionario Giuridico Simone 2001 = Nuovo Dizionario Giuridico con CD-ROM 2001. Napoli: Esselibri-Simone.

DRL 2003 = Tilch, Horst / Arloth, Frank (eds.) 2003: Deutsches Rechts-Lexikon. Ergänzungsband zur dritten Auflage. München: Beck.

DRL 2001 = Tilch, Horst / Arloth, Frank (eds.) 2001: Deutsches Rechts-Lexikon. In drei Bänden. München: Beck.

Enciclopedia Giuffrè 1958-2004 = Enciclopedia del diritto 1958-2004. Vol. I - Vol. XLVI. Milano: Giuffrè. 
Ferrara/Corsi 2006 = Ferrara, Francesco / Corsi, Francesco 2006: Gli imprenditori e le società. Milano: Giuffrè.

Galgano 2004 = Galgano, Francesco 2004: Diritto privato. Padova: CEDAM.

Galgano 1996 = Galgano, Francesco 1996: Dizionario enciclopedico del diritto. Vol. I: A-L, Vol. II: M-Z. Padova: CEDAM.

Garzantina Diritto 2001 = Enciclopedia Garzanti del Diritto 2001. Milano: Garzanti.

Hueck/Windbichler 2003 = Hueck, Götz / Windbichler, Christine 2003: Gesellschaftsrecht. Ein Studienbuch. München: Beck.

Kindler 2002 = Kindler, Peter 2002: Italienisches Handels- und Wirtschaftsrecht. Heidelberg: Recht und Wirtschaft.

Kindler 2007 = Kindler, Peter 2007: Einführung in das italienische Recht. Verfassungsrecht, Privatrecht und internationales Privatrecht. München: Beck.

Maganzi 2001 = Maganzi Gioeni d’Angiò, Francesca et al. 2001: Terminologisches Wörterbuch zum Schuldrecht italienisch / deutsch. Dizionario terminologico del diritto delle obbligazioni italiano / tedesco. München: Beck.

Musielak 2007 = Musielak, Hans-Joachim 2007: Grundkurs BGB. Eine Darstellung zur Vermittlung von Grundlagenwissen im bürgerlichen Recht mit Fällen und Fragen zur Lern- und Verständniskontrolle sowie mit Übungsklausuren. München: Beck.

\section{Übersetzerische Hilfsmittel (Datenbanken)}

BISTRO = Bozner Informationssystem für Rechtsterminologie der Europäischen Akademie Bozen: http://dev.eurac.edu:8080/cgi-bin/index/index.en.

JUSLEX = Datenbank für rechtssprachliche Terminologie und Phraseologie des Fachbereichs Übersetzen und Dolmetschen der Universität Bologna in Forlì (online nicht vor 2008).

TERMIT $=$ Terminologiedatenbank des Fachbereichs Übersetzen und Dolmetschen der Universität Triest: http://www.sslmit.units.it/termit/.

\section{Übersetzerische Hilfsmittel (Gesetzestexte)}

BGB = Bürgerliches Gesetzbuch: http://www.gesetze-im-internet.de/bundesrecht/bgb/ gesamt.pdf.

Codice Civile = Bartolini, Francesco (ed.) 2005: Il nuovo codice civile e le leggi complementari con CD-ROM. Piacenza: La Tribuna.

GmbHG = Gesetz betreffend die Gesellschaften mit beschränkter Haftung: http://www. gesetze-im-internet.de/bundesrecht/gmbhg/gesamt.pdf.

HGB = Handelsgesetzbuch: http://www.gesetze-im-internet.de/bundesrecht/hgb/gesamt.pdf.

\section{Sekundärliteratur}

Nord, Britta 2002: Hilfsmittel beim Übersetzen. Eine empirische Studie zum Rechercheverhalten professioneller Übersetzer. Frankfurt: Lang. 
Wiesmann, Eva 2004: Rechtsübersetzung und Hilfsmittel zur Translation. Wissenschaftliche Grundlagen und computergestützte Umsetzung eines lexikographischen Konzepts. Tübingen: Narr. 
